\title{
Alteration of pancreatic cancer cell functions by tumor-stromal cell interaction
}

\section{Shin Hamada *, Atsushi Masamune and Tooru Shimosegawa}

Division of Gastroenterology, Tohoku University Graduate School of Medicine, Sendai, Japan

\section{Edited by:}

Phoebe Phillips, University of New South Wales, Australia

Reviewed by:

Phoebe Phillips, University of New South Wales, Australia

Mert Erkan, Klinikum rechts der

Isar - Technische Universitaet

Muenchen, Germany

${ }^{*}$ Correspondence:

Shin Hamada, Division of Gastroenterology, Tohoku University Graduate School of Medicine, 1-1 Seiryo-machi Aobaku, Sendai,

Miyagi 980-8574, Japan

e-mail: hamadas@med.tohoku.ac.jp

Pancreatic cancer shows a characteristic tissue structure called desmoplasia, which consists of dense fibrotic stroma surrounding cancer cells. Interactions between pancreatic cancer cells and stromal cells promote invasive growth of cancer cells and establish a specific microenvironment such as hypoxia which further aggravates the malignant behavior of cancer cells. Pancreatic stellate cells (PSCs) play a pivotal role in the development of fibrosis within the pancreatic cancer tissue, and also affect cancer cell function. PSCs induce epithelial-mesenchymal transition and cancer stem cell (CSC)-related phenotypes in pancreatic cancer cells by activating multiple signaling pathways. In addition, pancreatic cancer cells and PSCs recruit myeloid-derived suppressor cells which attenuate the immune reaction against pancreatic cancer cells. As a result, pancreatic cancer cells become refractory against conventional therapies. The formation of the CSC-niche by stromal cells facilitates postoperative recurrence, re-growth of therapy-resistant tumors and distant metastasis. Conventional therapies targeting cancer cells alone have failed to conquer pancreatic cancer, but targeting the stromal cells and immune cells in animal experiments has provided evidence of improved therapeutic responses. A combination of novel strategies altering stromal cell functions could contribute to improving the pancreatic cancer prognosis.

Keywords: desmoplasia, pancreatic stellate cells, bone marrow derived cells, epithelial-mesenchymal transition, mast cells, cancer stem cells

\section{INTRODUCTION}

The prognosis of pancreatic cancer remains poor despite diagnostic and therapeutic improvements. A sensitive early detection screening method has not yet become available, and clinical symptoms such as obstructive jaundice or back pain are signs of advanced disease. Most patients are not eligible for curative surgery, due to the local invasion and distant metastasis (Hidalgo, 2010). Chemotherapy is an alternative treatment for patients with unresectable disease, but complete remission of pancreatic cancer by current regimens is rare. Postoperative recurrence is also frequent, presenting as liver metastasis or peritonitis carcinomatosa with a dismal prognosis (Hidalgo, 2010). In the United States, there were an estimated 45,220 new cases of pancreatic cancer in 2013 and more than $80 \%$ of those patients are expected to die from the disease, suggesting a critical state (Siegel et al., 2013). According to the Japan Pancreatic Cancer Registry, 3-year survival rate of pancreatic cancer was approximately 20\% (Egawa et al., 2012). These clinical outcomes are attributed to the aggressive nature of pancreatic cancer cells, which reveal invasive growth into surrounding organs and distant metastasis. Resistance against the conventional therapy such as chemotherapy or radiation is also a formidable

Abbreviations: ATRA, all-trans retinoic acid; CSCs, cancer stem cells; ECM, extracellular matrix; EMT, epithelial-mesenchymal transition; ERK, extracellular signal-regulated kinase; HIF1 $\alpha$, hypoxia-inducible factor 1 alpha; IL, interleukin; MAPK, mitogen-activated protein kinase; MDSCs, myeloid-derived suppressor cells; MMP3, matrix metalloproteinase 3; PSCs, pancreatic stellate cells; TGF- $\beta$, transforming growth factor- $\beta$; VEGF, vascular endothelial growth factor. problem in the clinical situation, but no radical solution has yet appeared.

Previously, these biological behaviors of pancreatic cancer cells were thought to result from cumulative gene mutations within the cancer cell itself. Major mutations in pancreatic cancer have been identified, such as an activating mutation of Kras, inactivating mutations of tumor suppressor p16, p53, and Smad4 (Hong et al., 2011). These mutations accumulate along with the increasing atypia of cells in the preneoplastic lesion, PanIN. However, the contribution of stromal cells during the pancreatic cancer progression is now widely recognized as playing crucial roles in pancreatic cancer cell survival, invasion, and metastasis. Pancreatic cancer shows a characteristic tissue structure called desmoplasia, which consists of dense fibrotic stroma surrounding the cancer cells (Erkan et al., 2012b). Recent research has revealed the involvement of a wide variety of host-derived normal cells in the development of the pancreatic cancer-specific tissue structure. Perpetuated inflammation caused by the unregulated growth of pancreatic cancer cells leads to the formation of desmoplasia, which could act as a physical barrier for the pancreatic cancer cells against the anti-cancer drugs and immune surveillance (Evans and Costello, 2012; Kozono et al., 2013; Tang et al., 2013). The activation of stromal cells by pancreatic cancer cells is a persisting event and involves multiple signaling pathways such as mitogen-activated protein kinase (MAPK) (Erkan et al., 2012a).

Pancreatic stellate cells (PSCs) reside within pancreatic parenchyma and remain in a quiescent state in normal pancreas. Quiescent PSCs contain vitamin A droplets in the cytoplasm 
(Masamune and Shimosegawa, 2009). Once activated by inflammation, PSCs start to proliferate and undergo myofibroblast-like phenotypic changes. Activated PSCs play a central role in pancreatic fibrosis by producing extracellular matrix (ECM) proteins and cytokines (Masamune and Shimosegawa, 2009, 2013). In addition to the residual PSCs, bone marrow-derived cells could also contribute to the population of PSCs (Masamune et al., 2009; Watanabe et al., 2009; Scarlett, 2013). After the bone marrow transplantation, bone marrow-derived cells accounted for $8.7 \%$ of PSCs in the pancreas. Induction of pancreatic fibrosis increased the bone marrow-derived cells up to $20 \%$ of activated PSCs (Watanabe et al., 2009). These bone marrow-derived cells were also capable of contributing to the desmoplastic stroma in a dimethylbenzanthracene-induced mouse pancreatic cancer model (Scarlett et al., 2011). These results suggest that the transdifferentiation of extrapancreatic cells leads to their involvement with pancreatic cancer cells as a stromal component. Furthermore, other types of cells could be recruited to the pancreatic cancer tissue. For example, pancreatic cancer induces the mobilization of myeloid-derived suppressor cells (MDSCs), which results in the recruitment of MDSCs within the tumor, leading to the attenuation of $\mathrm{CD}^{+} \mathrm{T}$-cell functions (Porembka et al., 2012). Local immunosuppression hampers the efficient elimination of cancer cells promoting disease progression. Other types of cells, such as mast cells, also accumulate within pancreatic cancer tissue, and activate PSCs (Ma et al., 2013).

Various lines of evidence suggest that the progression of pancreatic cancer requires interaction with host cells including stromal cells, inflammatory cells and immune cells. This tumorstromal interaction modifies multiple cell functions such as proliferation, invasion, survival, immune tolerance, and maintenance of the CSC function. The above mentioned cellular components and microenvironment of pancreatic cancer form a secure fortress for cancer cells by reinforcing each other. Since the establishment of pancreatic cancer requires more than 10 years, such interactions with pancreatic cancer cells should be a systemic phenomenon, not restricted to the diseased pancreas (Yachida et al., 2010). This review article focuses on the pancreatic cancer cells' functions affected by cell-to-cell interactions with various types of cells.

\section{TUMOR-STROMAL INTERACTION PROMOTES INVASIVE GROWTH OF PANCREATIC CANCER CELLS}

The tumor promoting role of pancreatic cancer stroma was first identified using PSCs isolated from surgically resected pancreatic cancer specimens. Subcutaneous co-injection of PSCs with human pancreatic cancer cell lines increased tumor growth (Bachem et al., 2005). Conditioned medium obtained from a culture of PSCs enhanced cell growth, invasion, migration and colony-formation of pancreatic cancer cells in vitro (Hwang et al., 2008). Co-injection of PSCs with human pancreatic cancer cell line BxPC3 in an orthotopic implantation model resulted in increased primary tumor size and metastatic foci, indicating the tumor-promoting role of PSCs in vivo (Hwang et al., 2008). Similar results were reported using another human pancreatic cancer cell line, MiaPaCa-2, describing that
PSC-conditioned medium enhanced cell growth and migration, but inhibited apoptosis (Vonlaufen et al., 2008). Coinjection of PSCs with cancer cells also increased the primary tumor size, regional invasion and distant metastasis (Vonlaufen et al., 2008). These results suggest that the interaction between PSCs and pancreatic cancer cells might affect the intracellular signal of pancreatic cancer cells. In a previous study, PSCconditioned medium activated MAPK and Akt pathways in pancreatic cancer cells, suggesting that PSCs are capable of mediating growth and survival-enhancing signals to cancer cells (Hwang et al., 2008).

Following these studies, the detailed mechanisms of invasive growth promotion by PSCs were extensively studied. PSCs expressing CD10 were able to promote the invasiveness of pancreatic cancer cells compared with $\mathrm{CD} 10^{-}$PSCs both in vitro and in vivo (Ikenaga et al., 2010). CD10 ${ }^{+}$PSCs produced higher amounts of matrix metalloproteinase 3 (MMP3) than $\mathrm{CD} 10^{-}$PSCs, whose siRNA-based knockdown attenuated the invasive capacity of the pancreatic cancer cells (Ikenaga et al., 2010). This study described that specific subpopulations of PSCs have distinct roles during the progression of pancreatic cancer. Another report described that PSCs resided within the metastatic nodules derived from the orthotopic implantation of pancreatic cancer cells with PSCs, suggesting that PSCs accompany cancer cells to the metastatic site, supporting colonization (Xu et al., 2010). This study confirmed the transendothelial migration of cancer cell-stimulated PSCs, which proved the capability of PSCs to extravasate to blood vessels (Xu et al., 2010). These cell functions of PSCs contribute to the establishment of an appropriate microenvironment for cancer cells to survive in the invaded or metastasized tissues.

The establishment of distant metastasis involves multiple processes, including epithelial-mesenchymal transition (EMT). EMT is characterized by the loss of epithelial phenotypes and gain of mesenchymal phenotypes, increased cellular migration and invasion, which is an indispensable process for the initiation of metastasis (Polyak and Weinberg, 2009). PSCs were found to trigger EMT in pancreatic cancer cells. Indirect co-culture of pancreatic cancer cells with PSCs induced EMT compatible phenotypic changes such as a fibroblast-like appearance and loose cell-to-cell contact in human pancreatic cancer cell lines Panc-1 and SUIT-2 (Kikuta et al., 2010). The expression of E-cadherin, cytokeratin 19, and membrane-associated betacatenin was decreased, while the expression of Vimentin and EMT-regulator Snail was increased. Along with the increased cellular migration, these phenotypic changes were considered to be compatible with EMT (Kikuta et al., 2010). In this study, blockade of a typical EMT-inducer, the transforming growth factor- $\beta$ (TGF- $\beta$ ) signal by anti-TGF- $\beta$-neutralizing antibody failed to attenuate EMT induction, excluding a central role of TGF- $\beta$ in this process. Therefore, the mediators of these tumor-promoting roles of PSCs remain elusive. The effect of direct cell-to-cell contact between cancer cells and stromal cells or mechanical stress from the tissue structure should be taken into account. Further study needs to be carried out to clarify the tumor-stromal interaction. 


\section{CANCER STEM CELL-RELATED PHENOTYPES ARE AFFECTED BY TUMOR-STROMAL INTERACTION}

Normal organs maintain tissue stem cells, which give rise to the various kinds of differentiated cells, to maintain the tissue structure. Cancer stem cells (CSCs) are the counterpart of the normal stem cells (Brabletz et al., 2009). In contrast to the normal stem cells, CSCs give rise to a wide variety of cancer cells with various degrees of differentiation and can reconstruct an entire population. CSCs also have self-renewal capacity, enabling them to survive after conventional therapy and leading to postoperative recurrence or re-growth of therapy-resistant tumors. Furthermore, EMT and increased cellular migration/invasion are some of the CSC-related phenotypes (Wellner et al., 2009). Together with the ability to reconstruct the entire cancer cell population, these phenotypes characterize: "migrating CSCs," which could initiate distant metastasis (Wellner et al., 2009). Until now, CSCs-containing cell fractions of pancreatic cancer cells were isolated using several cell surface markers such as $\mathrm{CD} 44^{+} \mathrm{CD} 24^{+} \mathrm{ESA}^{+}$cells or $\mathrm{CD} 133^{+}$cells (Hermann et al., 2007; Li et al., 2007). Though these cells are not identical with pure CSCs, they reveal resistance to chemotherapeutic agents, invasiveness, and self-renewal capacity. Theoretically, inhibition of CSCs' self-renewal could lead to curing pancreatic cancer. However, extracellular factors, which define the stemness of CSCs in pancreatic cancer, remain unclear. Since PSCs promote EMT in pancreatic cancer cells, the possible contribution of PSCs in the maintenance of stemness was assumed.

A recent report described that PSCs can enhance CSC-like phenotypes in pancreatic cancer cells in vitro, inducing the expression of the CSC-related genes ABCG2, Nestin, and LIN28 and increasing the spheroid formation in low-adhesion coated plates, also a feature of CSCs (Hamada et al., 2012). Co-injection of PSCs with pancreatic cancer cells also accelerated the subcutaneous tumor growth, suggesting the promotion of tumorigenicity in vivo (Hamada et al., 2012). Another study identified that PSCs form a niche for CSCs and promote self-renewal (Lonardo et al., 2012). In this study, Nodal-expressing pancreatic stellate cells could be an important component of the tumor stroma that supports pancreatic CSCs. These findings suggest the possibility of CSC-targeting strategies by inhibiting the cellular functions of PSCs. Conventional chemotherapy could expand the cancer cell population with the CSC phenotype, which leads to therapy-resistant tumors (Wang et al., 2009). The addition of PSC-inhibiting agents to conventional chemotherapy might be effective in reducing the therapy-resistant cancer cells.

\section{DESMOPLASIA PROVIDES HYPOXIC MICROENVIRONMENT}

Desmoplasia inhibits the perfusion of various molecules from blood by separating cancer cells from the blood vessels. Such a tissue structure also hampers oxygen perfusion, resulting in severe hypoxia within the tumor. The expression level of hypoxiainducible factor 1 alpha $(\mathrm{HIF} 1 \alpha)$ was correlated with the presence of a fibrotic focus in pancreatic cancer tissue, suggesting desmoplasia provides a hypoxic condition within the tumor (Couvelard et al., 2005). Exposure to a hypoxic condition stabilizes the transcriptional factor HIF $1 \alpha$ against proteasomal degradation, which induces HIF $1 \alpha$-target genes such as vascular endothelial growth factor (VEGF) or MMP3 (Duffy et al., 2003; Lin et al., 2008). In addition, hypoxia could induce profibrogenic and proangiogenic responses in PSCs, characterized by type I collagen expression and VEGF production (Masamune et al., 2008). These results indicate that the hypoxic condition derived from pancreatic fibrosis could be amplified by the feed-forward loop, nourishing further the hypoxic condition.

The hypoxic condition itself can exacerbate the malignant phenotype of pancreatic cancer cells. A previous report described that tumor hypoxia correlated with the metastasis of cancer cells in an orthotopic implantation model of pancreatic cancer (Buchler et al., 2004). The downstream target genes induced by hypoxia, VEGF and interleukin (IL)-6 enhance the invasive growth of pancreatic cancer cells (Bao et al., 2012). In addition to these conventional molecules, a novel class of molecules was recently identified. miRNA is a small, non-coding RNA that interacts with hundreds of target mRNAs, enabling the comprehensive regulation of cellular functions (Farazi et al., 2011). Among these miRNAs, miR-210 is specifically induced by hypoxia, and elevated expression of miR-210 is associated with poor survival in pancreatic cancer patients (Greither et al., 2010). Interestingly, PSCs are able to induce miR-210 expression in pancreatic cancer cells even under normoxic conditions via the extracellular signal-regulated kinase (ERK) and Akt pathways (Takikawa et al., 2013). This HIF $1 \alpha$-independent miR-210 induction affected the EMT of pancreatic cancer cells, suggesting an intriguing effect of tumor-stromal interaction.

Hypoxia also contributes to the CSC-related phenotypes of pancreatic cancer cells. Hypoxia induced the expression of the putative pancreatic cancer stem cell marker CD133 in human pancreatic cancer cell lines (Hashimoto et al., 2011). Besides CD133, the expression of CXC chemokine receptor 4 was also increased by hypoxia, and its expression was found in a highlyinvasive subpopulation of CSCs in another study (Hermann et al., 2007). In addition, the expression of other stem-cell markers, Nanog and Oct4, was increased by hypoxia in pancreatic cancer cells (Bao et al., 2012). Another study identified that HIF1 $\alpha$ inhibitor PX-478 sensitized pancreatic cancer cells to radiation (Schwartz et al., 2009). Since resistance to radiation is one of the CSC-related phenotypes, HIF1 $\alpha$-regulated signals might be involved in the maintenance of stemness. Together with the tumor-stromal interaction, hypoxia is thought to form a niche for CSCs.

\section{INTERACTION BETWEEN IMMUNE CELLS AND PANCREATIC CANCER CELLS}

Pancreatic cancer affects the host immune system to evade detection by immune surveillance. Examination of a mouse model of pancreatic cancer revealed leukocytic infiltration around the pre-invasive lesion of pancreatic cancer, consisting of MDSCs, immunosuppressive cells recruited from bone marrow (Clark et al., 2007). MDSCs are myeloid cells comprised of precursors of macrophages, dendritic cells, and granulocytes. The production of arginase, nitric oxide, and reactive oxygen species from MDSCs contributes to the suppression of T-cell functions (OstrandRosenberg and Sinha, 2009). These immune cells' interactions result in antigen-specific T-cell tolerance, a major mechanism 
of cancer cell escape from the host immune system (Wang, 2006). Detailed mechanisms by which pancreatic cancer affects the differentiation of myeloid cells remain controversial, but several markers of MDSCs [monocytic MDSC $\left(\mathrm{CD} 11 \mathrm{~b}^{+} \mathrm{CD}_{14}^{+}\right)$ or granulocytic MDSC $\left.\left(\mathrm{CD} 11 \mathrm{~b}^{+} \mathrm{CD} 15^{+}\right)\right]$have been reported (Goedegebuure et al., 2011).

The degree of MDSCs' induction has an impact on pancreatic cancer progression, and therefore affects the clinical outcomes. For example, the amount of the peripheral blood MDSCs could correlate with a patient's prognosis. MDSCs were significantly elevated in blood samples from cancer-bearing patients including pancreatic cancer, and the MDSC level was found to be an independent prognostic factor for patient survival (Gabitass et al., 2011). This report also described the elevation of Th2 cytokine IL-13 in a blood sample, which correlated with the MDSC level. Another report described that MDSCs are related to chronic inflammation in cancer-bearing patients, leading to the deterioration of the nutritional status (Ohki et al., 2012). The MDSC level was inversely correlated with the serum concentration of total protein, suggesting a systemic effect of tumor-induced MDSCs. These observations suggest that the general condition of pancreatic cancer-bearing patients is modulated by the altered immune reaction, which results from the interaction between pancreatic cancer cells and immune cells.

\section{PSCs' CONTRIBUTION TO THE ALTERATION OF IMMUNE CELL FUNCTIONS}

In addition to pancreatic cancer cells, PSCs also modify the immune cell functions. Pancreatic cancer-associated PSCs were found to promote the differentiation of myeloid-derived cells to MDSCs through the STAT3 pathway (Mace et al., 2013). The induction of MDSCs from peripheral blood mononuclear cells by PSC-conditioned medium was mediated by IL-6. MDSCs induced by PSC-conditioned medium showed CD11b expression and inhibited autologous T-cell proliferation. Targeting this signaling pathway abrogated the PSC-conditioned medium-induced MDSC differentiation, suggesting the possibility of a novel therapy by inhibiting the induction of MDSCs. Indeed, suppressing the functions of MDSCs by synthetic triterpenoid successfully attenuated the tumor growth in mice through the restoration of the immune reaction against cancer cells (Nagaraj et al., 2010). These lines of evidence suggest that the differentiation and recruitment of MDSCs into pancreatic cancer could be promising therapeutic targets.

Furthermore, a recent report described that activated PSCs sequester $\mathrm{CD}^{+}$T-cells and reduce infiltration around the tumor, resulting in a reduced anti-tumor immune response (Ene-Obong et al., 2013). The degree of $\mathrm{CD}^{+}$T-cell infiltration around the tumor positively correlated with patients' survival, indicating the contribution of the host immune reaction to inhibiting the progression of pancreatic cancer (Ene-Obong et al., 2013). A similar sequestration of $\mathrm{CD}^{+}$T-cells was observed in a genetically-engineered mouse model of pancreatic cancer, KPC mice (Ene-Obong et al., 2013). This mouse model develops invasive pancreatic cancer based on the conditional expression of mutant $\mathrm{K}$-ras (constitutively active mutation G12D) and mutant p53 (inactivating mutation $\mathrm{R} 172 \mathrm{H}$ ) in the pancreas, recapitulating human pancreatic cancer including prominent desmoplasia (Hingorani et al., 2005). All-trans retinoic acid (ATRA) administration to KPC mice attenuated PSC activation in KPC mice, which resulted in the restoration of $\mathrm{CD}^{+} \mathrm{T}$ cell infiltration around the pancreatic cancer cells. Interestingly, PSCs-derived CXCL12 increased the chemotaxis of CD8 ${ }^{+}$T-cells toward PSCs, the knockdown of which showed an identical effect with ATRA (Ene-Obong et al., 2013). Inhibition of CXCL12 and ATRA might become a novel therapeutic option to restore the immune response against pancreatic cancer.

PSCs interact with another type of immune cells, which also promote the growth of pancreatic cancer. Mast cells trigger type I hypersensitivity in various diseases such as bronchial asthma and urticaria (Beunk et al., 2013). The accumulation of mast cells within pancreatic cancer was found in the pancreatic tumor of a mouse model that conditionally expresses constitutively active K-ras (G12V) in pancreas (Chang et al., 2011). This study confirmed the important role of mast cells in the pancreatic cancer progression using an orthotopic implantation model. Pancreatic tumor growth in mast cell-deficient Kit (w-sh/w-sh) mice that received orthotopic implantation of pancreatic cancer cells was suppressed, and the reconstitution of mast cells from wild-type bone marrow aggravated the orthotopic tumor growth (Chang et al., 2011). These results revealed a novel role of mast cells as promoters of pancreatic cancer. Detailed mechanisms of the role of mast cells in promoting cancer were examined thereafter. Pancreatic cancer cell-conditioned medium promoted mast cell migration and co-culture of mast cells with pancreatic cancer cells or PSCs stimulated mast cell activation, confirmed by the release of tryptase and tumor necrosis factor- $\alpha$ (Ma et al., 2013). Mast cells promoted the proliferation of PSCs and mast cell-derived IL-13 mediated this process. Interestingly, the IL-13 elevation was also correlated with the increase of MDSCs, suggesting possible cross-talk. Recent research noted that mast cells can enhance the immunosuppressive functions of MDSCs, supporting this speculation (Saleem et al., 2012). Taken together, mast cells contribute to pancreatic cancer progression by increasing cell proliferation and activating PSCs. Targeting mast cells for pancreatic cancer treatment has not yet been evaluated, but this strategy might yield an additional way to suppress the stromal reaction and immunosuppression in pancreatic cancer.

\section{TARGETING TUMOR-STROMAL INTERACTION; POSSIBILITY AND PROBLEMS}

As summarized in this review, tumor-stromal interaction has pivotal role in pancreatic cancer progression. Interventions directed to the desmoplasia-inducing signaling pathways revealed favorable effects, such as the inhibition of Shh pathway or CTGF pathway in mouse model (Olive et al., 2009; Neesse et al., 2013). However, the phase II clinical trial of Shh inhibitor IPI-926 halted due to the significantly shorter survival in patients on the gemcitabine plus IPI-926 arm. This result might be due to the heterogeneity of human disease, distinct from the relatively homogeneous nature of tumors in the mouse model, arising from uniform genetic background. It is also possible that simple depletion of fibrotic stroma promotes cancer cell spread. To conquer these discrepancies between human disease and mouse model, 


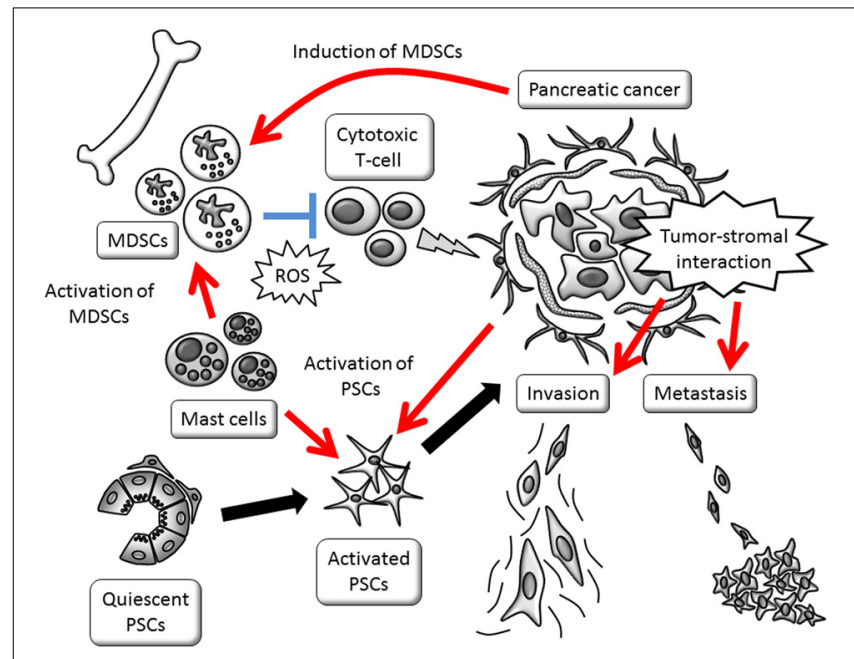

FIGURE 1 | Schematic view of tumor-stromal interactions affecting invasive growth and escape from immune surveillance. Promotion of invasive phenotype is mainly provided by activated PSCs. PSC activation and MDSC induction are stimulated by cancer cells. MDSCs, myeloid-derived suppressor cells; PSCs, pancreatic stellate cells; ROS, reactive oxygen species.

detailed examination of clinical samples and further understanding about the diversity of human disease are required.

Targeting immune cells could be an alternative approach to modify the tumor microenvironment. Restoring the immune reaction could be performed by several agents, such as ATRA or IL-12 in mouse model, which exhibited therapeutic effects (Kerkar et al., 2011; Ene-Obong et al., 2013). ATRA was also used to induce differentiation of MDSCs in patients with metastatic renal cell carcinoma, without significant toxicity (Mirza et al., 2006). Use of these agents in combination with conventional chemotherapy could have some benefits that needs to be evaluated by further study.

\section{CONCLUSION}

Pancreatic cancer is a deadly disease and its establishment proceeds silently. Stromal reaction and escape from immune surveillance are perpetuating systemic changes. The involvement of stromal cell activation and immunosuppression enable cancer cells to remain in a favorable niche, leading to distant metastasis and therapy resistance. A schematic view of the cancer cell-stromal cell interaction is shown in Figure 1. Conventional strategies that target the cancer cells themselves failed to cure pancreatic cancer, due to the evolution in cancer cells and protection by the tumor stroma. However, tumor stromal cells originally maintain normal signaling pathways and relatively homogenous characteristics, showing better therapeutic responses than pancreatic cancer cells. Among these types of cells, PSCs show multifaceted roles in modulating cell-to-cell interactions. PSCs' contribution to the multiple interactions is summarized in Figure 2. Targeting PSCs, immune cells and the tumor stromal structure could be alternative therapies for pancreatic cancer. Further study is required to improve the clinical outcomes of pancreatic cancer patients.

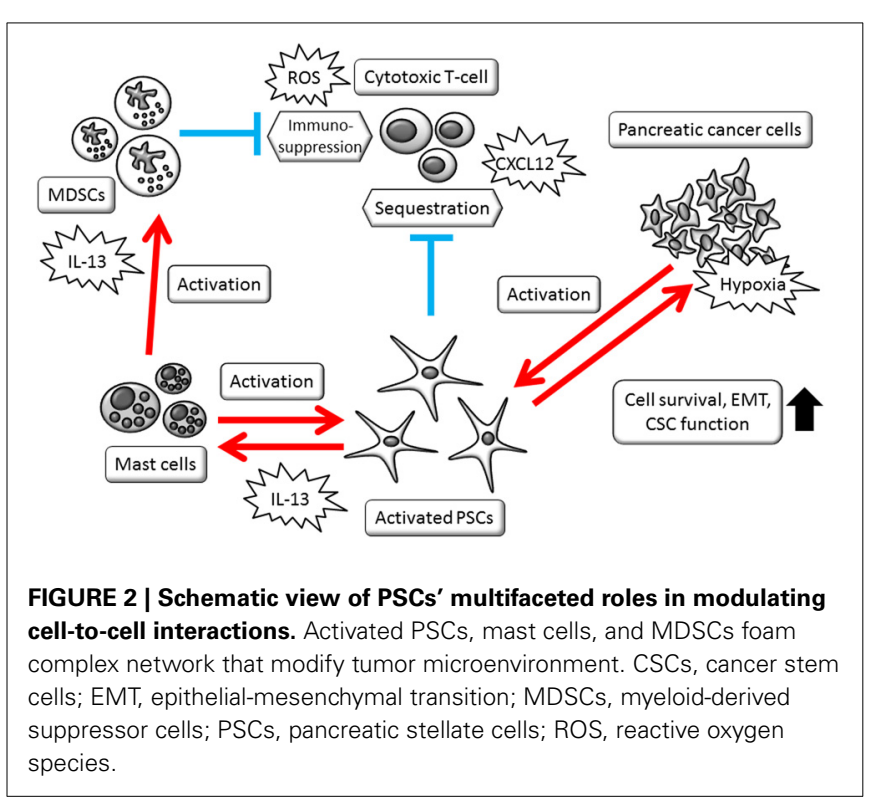

\section{ACKNOWLEDGMENTS}

This work was supported in part by Grant-in-Aid from the Japan Society for the Promotion of Science (23591008, 24790674, and 23390194) and by the Research Committee of Intractable Pancreatic Diseases (Principal investigator: Tooru Shimosegawa) provided by the Ministry of Health, Labor and Welfare of Japan.

\section{REFERENCES}

Bachem, M. G., Schunemann, M., Ramadani, M., Siech, M., Beger, H., Buck, A., et al. (2005). Pancreatic carcinoma cells induce fibrosis by stimulating proliferation and matrix synthesis of stellate cells. Gastroenterology 128, 907-921. doi: 10.1053/j.gastro.2004.12.036

Bao, B., Ali, S., Ahmad, A., Azmi, A. S., Li, Y., Banerjee, S., et al. (2012). Hypoxiainduced aggressiveness of pancreatic cancer cells is due to increased expression of VEGF, IL-6 and miR-21, which can be attenuated by CDF treatment. PLoS ONE 7:e50165. doi: 10.1371/journal.pone.0050165

Beunk, L., Verwoerd, A., van Overveld, F. J., and Rijkers, G. T. (2013). Role of mast cells in mucosal diseases: current concepts and strategies for treatment. Expert Rev. Clin. Immunol. 9, 53-63. doi: 10.1586/eci.12.82

Brabletz, S., Schmalhofer, O., and Brabletz, T. (2009). Gastrointestinal stem cells in development and cancer. J. Pathol. 217, 307-317. doi: 10.1002/path.2475

Buchler, P., Reber, H. A., Lavey, R. S., Tomlinson, J., Buchler, M. W., Friess, H., et al. (2004). Tumor hypoxia correlates with metastatic tumor growth of pancreatic cancer in an orthotopic murine model. J. Surg. Res. 120, 295-303. doi: 10.1016/j.jss.2004.02.014

Chang, D. Z., Ma, Y., Ji, B., Wang, H., Deng, D., Liu, Y., et al. (2011). Mast cells in tumor microenvironment promotes the in vivo growth of pancreatic ductal adenocarcinoma. Clin. Cancer Res. 17, 7015-7023. doi: 10.1158/10780432.CCR-11-0607

Clark, C. E., Hingorani, S. R., Mick, R., Combs, C., Tuveson, D. A., and Vonderheide, R. H. (2007). Dynamics of the immune reaction to pancreatic cancer from inception to invasion. Cancer Res. 67, 9518-9527. doi: 10.1158/00085472.CAN-07-0175

Couvelard, A., O’Toole, D., Leek, R., Turley, H., Sauvanet, A., Degott, C., et al. (2005). Expression of hypoxia-inducible factors is correlated with the presence of a fibrotic focus and angiogenesis in pancreatic ductal adenocarcinomas. Histopathology 46, 668-676. doi: 10.1111/j.1365-2559.2005.02160.x

Duffy, J. P., Eibl, G., Reber, H. A., and Hines, O. J. (2003). Influence of hypoxia and neoangiogenesis on the growth of pancreatic cancer. Mol. Cancer 2, 12. doi: $10.1186 / 1476-4598-2-12$ 
Egawa, S., Toma, H., Ohigashi, H., Okusaka, T., Nakao, A., Hatori, T., et al. (2012). Japan pancreatic cancer registry; 30th year anniversary: japan pancreas society. Pancreas 41, 985-992. doi: 10.1097/MPA.0b013e318258055c

Ene-Obong, A., Clear, A. J., Watt, J., Wang, J., Fatah, R., Riches, J. C., et al. (2013). Activated pancreatic stellate cells sequester $\mathrm{CD} 8^{+} \mathrm{T}$-Cells to reduce their infiltration of the juxtatumoral compartment of pancreatic ductal adenocarcinoma. Gastroenterology. doi: 10.1053/j.gastro.2013.07.025. [Epub ahead of print].

Erkan, M., Adler, G., Apte, M. V., Bachem, M. G., Buchholz, M., Detlefsen, S., et al. (2012b). StellaTUM: current consensus and discussion on pancreatic stellate cell research. Gut 61, 172-178. doi: 10.1136/gutjnl-2011-301220

Erkan, M., Hausmann, S., Michalski, C. W., Fingerle, A. A., Dobritz, M., Kleeff, J., et al. (2012a). The role of stroma in pancreatic cancer: diagnostic and therapeutic implications. Nat. Rev. Gastroenterol. Hepatol. 9, 454-467. doi: $10.1038 /$ nrgastro.2012.115

Evans, A., and Costello, E. (2012). The role of inflammatory cells in fostering pancreatic cancer cell growth and invasion. Front. Physiol. 3:270. doi: 10.3389/fphys.2012.00270

Farazi, T. A., Spitzer, J. I., Morozov, P., and Tuschl, T. (2011). miRNAs in human cancer. J. Pathol. 223, 102-115. doi: 10.1002/path.2806

Gabitass, R. F., Annels, N. E., Stocken, D. D., Pandha, H. A., and Middleton, G. W. (2011). Elevated myeloid-derived suppressor cells in pancreatic, esophageal and gastric cancer are an independent prognostic factor and are associated with significant elevation of the Th2 cytokine interleukin-13. Cancer Immunol. Immunother. 60, 1419-1430. doi: 10.1007/s00262-011-1028-0

Goedegebuure, P., Mitchem, J. B., Porembka, M. R., Tan, M. C., Belt, B. A., Wang-Gillam, A., et al. (2011). Myeloid-derived suppressor cells: general characteristics and relevance to clinical management of pancreatic cancer. Curr. Cancer Drug Targets 11, 734-751. doi: 10.2174/156800911796191024

Greither, T., Grochola, L. F., Udelnow, A., Lautenschlager, C., Wurl, P., and Taubert, H. (2010). Elevated expression of microRNAs 155, 203, 210 and 222 in pancreatic tumors is associated with poorer survival. Int. J. Cancer 126, 73-80. doi: $10.1002 /$ ijc. 24687

Hamada, S., Masamune, A., Takikawa, T., Suzuki, N., Kikuta, K., Hirota, M., et al. (2012). Pancreatic stellate cells enhance stem cell-like phenotypes in pancreatic cancer cells. Biochem. Biophys. Res. Commun. 421, 349-354. doi: 10.1016/j.bbrc.2012.04.014

Hashimoto, O., Shimizu, K., Semba, S., Chiba, S., Ku, Y., Yokozaki, H., et al. (2011). Hypoxia induces tumor aggressiveness and the expansion of CD133-positive cells in a hypoxia-inducible factor-1alpha-dependent manner in pancreatic cancer cells. Pathobiology 78, 181-192. doi: 10.1159/000325538

Hermann, P. C., Huber, S. L., Herrler, T., Aicher, A., Ellwart, J. W., Guba, M., et al. (2007). Distinct populations of cancer stem cells determine tumor growth and metastatic activity in human pancreatic cancer. Cell Stem Cell 1, 313-323. doi: 10.1016/j.stem.2007.06.002

Hidalgo, M. (2010). Pancreatic cancer. N. Engl. J. Med. 362, 1605-1617. doi: 10.1056/NEJMra0901557

Hingorani, S. R., Wang, L., Multani, A. S., Combs, C., Deramaudt, T. B., Hruban, R. H., et al. (2005). Trp53R172H and KrasG12D cooperate to promote chromosomal instability and widely metastatic pancreatic ductal adenocarcinoma in mice. Cancer Cell 7, 469-483. doi: 10.1016/j.ccr.2005. 04.023

Hong, S. M., Park, J. Y., Hruban, R. H., and Goggins, M. (2011). Molecular signatures of pancreatic cancer. Arch. Pathol. Lab Med. 135, 716-727. doi: 10.1043/2010-0566-RA.1

Hwang, R. F., Moore, T., Arumugam, T., Ramachandran, V., Amos, K. D., Rivera, A., et al. (2008). Cancer-associated stromal fibroblasts promote pancreatic tumor progression. Cancer Res. 68, 918-926. doi: 10.1158/0008-5472. CAN-07-5714

Ikenaga, N., Ohuchida, K., Mizumoto, K., Cui, L., Kayashima, T., Morimatsu, K., et al. (2010). CD10 ${ }^{+}$pancreatic stellate cells enhance the progression of pancreatic cancer. Gastroenterology 139, 1041-1051. doi: 10.1053/j.gastro.2010.05.084

Kerkar, S. P., Goldszmid, R. S., Muranski, P., Chinnasamy, D., Yu, Z., Reger, R. N., et al. (2011). IL-12 triggers a programmatic change in dysfunctional myeloid-derived cells within mouse tumors. J. Clin. Invest. 121, 4746-4757. doi: 10.1172/JCI58814

Kikuta, K., Masamune, A., Watanabe, T., Ariga, H., Itoh, H., Hamada, S., et al. (2010). Pancreatic stellate cells promote epithelial-mesenchymal transition in pancreatic cancer cells. Biochem. Biophys. Res. Commun. 403, 380-384. doi: 10.1016/j.bbrc.2010.11.040
Kozono, S., Ohuchida, K., Eguchi, D., Ikenaga, N., Fujiwara, K., Cui, L., et al. (2013). Pirfenidone inhibits pancreatic cancer desmoplasia by regulating stellate cells. Cancer Res. 73, 2345-2356. doi: 10.1158/0008-5472.CAN-12-3180

Li, C., Heidt, D. G., Dalerba, P., Burant, C. F., Zhang, L., Adsay, V., et al. (2007). Identification of pancreatic cancer stem cells. Cancer Res. 67, 1030-1037. doi: 10.1158/0008-5472.CAN-06-2030

Lin, J. L., Wang, M. J., Lee, D., Liang, C. C., and Lin, S. (2008). Hypoxiainducible factor-1alpha regulates matrix metalloproteinase- 1 activity in human bone marrow-derived mesenchymal stem cells. FEBS Lett. 582, 2615-2619. doi: 10.1016/j.febslet.2008.06.033

Lonardo, E., Frias-Aldeguer, J., Hermann, P. C., and Heeschen, C. (2012). Pancreatic stellate cells form a niche for cancer stem cells and promote their self-renewal and invasiveness. Cell Cycle 11, 1282-1290. doi: 10.4161/cc.19679

Ma, Y., Hwang, R. F., Logsdon, C. D., and Ullrich, S. E. (2013). Dynamic mast cellstromal cell interactions promote growth of pancreatic cancer. Cancer Res. 73, 3927-3937. doi: 10.1158/0008-5472.CAN-12-4479

Mace, T. A., Ameen, Z., Collins, A., Wojcik, S., Mair, M., Young, G. S., et al. (2013). Pancreatic cancer-associated stellate cells promote differentiation of myeloid-derived suppressor cells in a STAT3-dependent manner. Cancer Res. 73, 3007-3018. doi: 10.1158/0008-5472.CAN-12-4601

Masamune, A., Kikuta, K., Watanabe, T., Satoh, K., Hirota, M., and Shimosegawa, T. (2008). Hypoxia stimulates pancreatic stellate cells to induce fibrosis and angiogenesis in pancreatic cancer. Am. J. Physiol. Gastrointest. Liver Physiol. 295 G709-G717. doi: 10.1152/ajpgi.90356.2008

Masamune, A., and Shimosegawa, T. (2009). Signal transduction in pancreatic stellate cells. J. Gastroenterol. 44, 249-260. doi: 10.1007/s00535-009-0013-2

Masamune, A., and Shimosegawa, T. (2013). Pancreatic stellate cellsmulti-functional cells in the pancreas. Pancreatology 13, 102-105. doi: 10.1016/j.pan.2012.12.058

Masamune, A., Watanabe, T., Kikuta, K., and Shimosegawa, T. (2009). Roles of pancreatic stellate cells in pancreatic inflammation and fibrosis. Clin. Gastroenterol. Hepatol. 7, S48-S54. doi: 10.1016/j.cgh.2009.07.038

Mirza, N., Fishman, M., Fricke, I., Dunn, M., Neuger, A. M., Frost, T. J., et al. (2006). All-trans-retinoic acid improves differentiation of myeloid cells and immune response in cancer patients. Cancer Res. 66, 9299-9307. doi: 10.1158/0008-5472.CAN-06-1690

Nagaraj, S., Youn, J. I., Weber, H., Iclozan, C., Lu, L., Cotter, M. J., et al. (2010). Antiinflammatory triterpenoid blocks immune suppressive function of MDSCs and improves immune response in cancer. Clin. Cancer Res. 16, 1812-1823. doi: 10.1158/1078-0432.CCR-09-3272

Neesse, A., Frese, K. K., Bapiro, T. E., Nakagawa, T., Sternlicht, M. D., Seeley, T. W., et al. (2013). CTGF antagonism with mAb FG-3019 enhances chemotherapy response without increasing drug delivery in murine ductal pancreas cancer. Proc. Natl. Acad. Sci. U.S.A. 110, 12325-12330. doi: 10.1073/pnas.1300415110

Ohki, S., Shibata, M., Gonda, K., Machida, T., Shimura, T., Nakamura, I., et al. (2012). Circulating myeloid-derived suppressor cells are increased and correlate to immune suppression, inflammation and hypoproteinemia in patients with cancer. Oncol. Rep. 28, 453-458. doi: 10.3892/or.2012.1812

Olive, K. P., Jacobetz, M. A., Davidson, C. J., Gopinathan, A., McIntyre, D., Honess, D., et al. (2009). Inhibition of Hedgehog signaling enhances delivery of chemotherapy in a mouse model of pancreatic cancer. Science 324, 1457-1461. doi: $10.1126 /$ science. 1171362

Ostrand-Rosenberg, S., and Sinha, P. (2009). Myeloid-derived suppressor cells: linking inflammation and cancer. J. Immunol. 182, 4499-4506. doi: 10.4049/jimmunol.0802740

Polyak, K., and Weinberg, R. A. (2009). Transitions between epithelial and mesenchymal states: acquisition of malignant and stem cell traits. Nat. Rev. Cancer 9, 265-273. doi: 10.1038/nrc2620

Porembka, M. R., Mitchem, J. B., Belt, B. A., Hsieh, C. S., Lee, H. M., Herndon, J., et al. (2012). Pancreatic adenocarcinoma induces bone marrow mobilization of myeloid-derived suppressor cells which promote primary tumor growth. Cancer Immunol. Immunother. 61, 1373-1385. doi: 10.1007/s00262-011-1178-0

Saleem, S. J., Martin, R. K., Morales, J. K., Sturgill, J. L., Gibb, D. R., Graham, L., et al. (2012). Cutting edge: mast cells critically augment myeloid-derived suppressor cell activity. J. Immunol. 189, 511-515. doi: 10.4049/jimmunol.1200647

Scarlett, C. J. (2013). Contribution of bone marrow derived cells to the pancreatic tumor microenvironment. Front. Physiol. 4:56. doi: 10.3389/fphys.2013.00056

Scarlett, C. J., Colvin, E. K., Pinese, M., Chang, D. K., Morey, A. L., Musgrove, E. A., et al. (2011). Recruitment and activation of pancreatic stellate cells from the 
bone marrow in pancreatic cancer: a model of tumor-host interaction. PLoS ONE 6:e26088. doi: 10.1371/journal.pone.0026088

Schwartz, D. L., Powis, G., Thitai-Kumar, A., He, Y., Bankson, J., Williams, R., et al. (2009). The selective hypoxia inducible factor-1 inhibitor PX-478 provides in vivo radiosensitization through tumor stromal effects. Mol. Cancer Ther. 8, 947-958. doi: 10.1158/1535-7163.MCT-08-0981

Siegel, R., Naishadham, D., and Jemal, A. (2013). Cancer statistics 2013. CA Cancer J. Clin. 63, 11-30. doi: 10.3322/caac.21166

Takikawa, T., Masamune, A., Hamada, S., Nakano, E., Yoshida, N., and Shimosegawa, T. (2013). miR-210 regulates the interaction between pancreatic cancer cells and stellate cells. Biochem. Biophys. Res. Commun. 437, 433-439. doi: 10.1016/j.bbrc.2013.06.097

Tang, D., Wang, D., Yuan, Z., Xue, X., Zhang, Y., An, Y., et al. (2013). Persistent activation of pancreatic stellate cells creates a microenvironment favorable for the malignant behavior of pancreatic ductal adenocarcinoma. Int. J. Cancer 132, 993-1003. doi: 10.1002/ijc.27715

Vonlaufen, A., Joshi, S., Qu, C., Phillips, P. A., Xu, Z., Parker, N. R., et al. (2008). Pancreatic stellate cells: partners in crime with pancreatic cancer cells. Cancer Res. 68, 2085-2093. doi: 10.1158/0008-5472.CAN-07-2477

Wang, R. F. (2006). Immune suppression by tumor-specific CD4 ${ }^{+}$ regulatory T-cells in cancer. Semin. Cancer Biol. 16, 73-79. doi: 10.1016/j.semcancer.2005.07.009

Wang, Z., Li, Y., Kong, D., Banerjee, S., Ahmad, A., Azmi, A. S., et al. (2009). Acquisition of epithelial-mesenchymal transition phenotype of gemcitabineresistant pancreatic cancer cells is linked with activation of the notch signaling pathway. Cancer Res. 69, 2400-2407. doi: 10.1158/0008-5472.CAN-08-4312

Watanabe, T., Masamune, A., Kikuta, K., Hirota, M., Kume, K., Satoh, K., et al. (2009). Bone marrow contributes to the population of pancreatic stellate cells in mice. Am. J. Physiol. Gastrointest. Liver Physiol. 297, G1138-G1146. doi: 10.1152/ajpgi.00123.2009
Wellner, U., Schubert, J., Burk, U. C., Schmalhofer, O., Zhu, F., Sonntag, A., et al. (2009). The EMT-activator ZEB1 promotes tumorigenicity by repressing stemness-inhibiting microRNAs. Nat. Cell. Biol. 11, 1487-1495. doi: $10.1038 /$ ncb 1998

Xu, Z., Vonlaufen, A., Phillips, P. A., Fiala-Beer, E., Zhang, X., Yang, L., et al. (2010). Role of pancreatic stellate cells in pancreatic cancer metastasis. Am. J. Pathol. 177, 2585-2596. doi: 10.2353/ajpath.2010. 090899

Yachida, S., Jones, S., Bozic, I., Antal, T., Leary, R., Fu, B., et al. (2010). Distant metastasis occurs late during the genetic evolution of pancreatic cancer. Nature 467, 1114-1117. doi: 10.1038/nature09515

Conflict of Interest Statement: The authors declare that the research was conducted in the absence of any commercial or financial relationships that could be construed as a potential conflict of interest.

Received: 09 September 2013; paper pending published: 01 October 2013; accepted: 14 October 2013; published online: 01 November 2013.

Citation: Hamada S, Masamune A and Shimosegawa T (2013) Alteration of pancreatic cancer cell functions by tumor-stromal cell interaction. Front. Physiol. 4:318. doi: 10.3389/fphys.2013.00318

This article was submitted to Gastrointestinal Sciences, a section of the journal Frontiers in Physiology.

Copyright (c) 2013 Hamada, Masamune and Shimosegawa. This is an openaccess article distributed under the terms of the Creative Commons Attribution License (CC BY). The use, distribution or reproduction in other forums is permitted, provided the original author(s) or licensor are credited and that the original publication in this journal is cited, in accordance with accepted academic practice. No use, distribution or reproduction is permitted which does not comply with these terms. 